Review

\title{
Posaconazole: A Review of Drug Interactions with HIV Antiretroviral Agents
}

\author{
Mara Poulakos ${ }^{1}$, Amy Henneman ${ }^{1, *}$ and Simon Leung ${ }^{2}$
}

1 Lloyd L. Gregory School of Pharmacy, Palm Beach Atlantic University, 901 South Flagler Drive, West Palm Beach, FL 33416, USA; E-Mail: mara_poulakos@pba.edu

2 Department of Pharmacy Services, Memorial Regional Hospital, 3501 Johnson Street, Hollywood, FL 33021, USA; E-Mail: sleung@mhs.net

* Author to whom correspondence should be addressed; E-Mail: amy_henneman@pba.edu; Tel.: +1-561-803-2721; Fax: +1-561-803-2703.

Received: 5 December 2013; in revised form: 14 February 2014 / Accepted: 20 February 2014 / Published: 4 March 2014

\begin{abstract}
The purpose of this review is to examine the literature for reports of clinically significant interactions noted amongst HIV antiretroviral medications when coadministered with posaconazole. A literature search was conducted to identify studies addressing drug interactions between posaconazole and HIV antiretroviral medications. Two pharmacokinetic studies and three clinical trials involving the administration of posaconazole to HIV-infected patients were identified. The pharmacokinetic studies involved concomitant administration of either a protease inhibitor (PI) or non-nucleoside reverse transcriptase inhibitor (NNRTI). Both studies showed alterations in systemic concentrations of either posaconazole or the HIV antiretroviral when administered together. Of the three clinical trials, all patients were on HIV antiretrovirals. However, their potential interaction with posaconazole was not explored. To date, there is no published literature regarding the interaction between maraviroc or elvitegravir and posaconazole. Dose adjustments for each are recommended when coadministered with strong CYP 3A4 inhibitors or inducers. Currently available literature points to the potential for clinically significant drug interactions when posaconazole is coadministered with HIV antiretrovirals, specifically NNRTIs and PIs. More studies are needed involving a wider range of HIV antiretrovirals to determine the significance of the interaction. Clinicians should be aware of this potentially significant interaction and avoid concomitant administration when possible. When available, consideration should be given to therapeutic drug monitoring of antiretroviral serum concentrations in select patients.
\end{abstract}


Keywords: posaconazole; HIV antiretroviral; drug interaction

\section{Introduction}

Posaconazole is an extended spectrum triazole antifungal agent that is structurally similar to itraconazole. It has received Food and Drug Administration (FDA) approval for use in the prophylaxis of invasive fungal infections, specifically Aspergillus and Candida infections, in high risk immunocompromised patients who are 13 years or older. It is also indicated for the treatment of oropharyngeal candidiasis, including oropharyngeal candidiasis refractory to itraconazole and/or fluconazole [1]. In addition, limited information has suggested that posaconazole may be useful in the treatment of fungal infections of the central nervous system [2]. Posaconazole exerts its activity by inhibiting fungal ergosterol biosynthesis through binding to the heme factor situated in the target site of the cytochrome P450 (CYP450) dependent enzyme lanosterol 14 $\alpha$-demethylase [3,4]. Posaconazole has fungicidal activity against Cryptococcus neoformans, Aspergillus species (i.e., A. fumigatus, A. flavus and A. terreus), Blastomyces dermatitidis, Trichosporon and specific Candida species (i.e., C. krusei, C. parapsilosis, C. lusitaniae and C. inconspicua). It has also shown fungistatic activity against most other Candida species, Scedosporium Coccidiodes, Zygomycetes and specific Fusarium species (i.e., F. oxysporum and F. monilforme) [5,6]. Guidelines on the management of opportunistic infections in HIV-infected adults and adolescents currently recommend posaconazole as a first line option for refractory oral or esophageal candidiasis in patients who have received multiple courses of azole antifungals. Posaconazole is recommended as an alternative treatment option for patients who cannot take fluconazole for oral candidiasis as well as an option for patients who have failed first-line treatment options for histoplasmosis and aspergillosis, though data in this patient population is lacking [7].

Available as an oral suspension and delayed-release tablet commercially, the gastrointestinal absorption of posaconazole is significantly enhanced when coadministered with food, particularly high fat meals [8-14]. Oral absorption of posaconazole is $\mathrm{pH}$ dependent, its concentrations increase when coadministered with an acidic carbonated beverage or nutritional supplement if the patient is unable to eat a full meal, whereas its concentrations decrease in a basic gastric environment [13]. It is recommended that a patient consume a full fatty meal when taking the oral suspension. Although the new delayed-release tablets provide higher serum concentrations in the fasting state than the oral suspension, it is recommended that tablets be administered with food when possible [1]. Concomitant administration of posaconazole oral suspension and cimetidine or esomeprazole should be avoided due to decreased posaconazole plasma concentrations. Concomitant administration of delayed-release posaconazole tablets with antacids, histamine 2 receptor antagonists, or proton pump inhibitors has shown no clinically relevant effects on posaconazole absorption [1]. Posaconazole has a relatively long absorption phase $(\sim 5 \mathrm{~h})$, large volume of distribution $(>834 \mathrm{~L})$, which is dose dependent, and a high plasma protein binding ( $>98.5 \%$ ) regardless of the degree of renal function and hemodialysis [8,15-17]. The mean systemic exposure value of posaconazole oral suspension for the single dose phase (AUC $\mathrm{A}_{0-\infty}$, $\mathrm{mcg} \cdot \mathrm{h} / \mathrm{mL})$ is 18.4 whereas for the multiple dosing phase $\left(\mathrm{AUC}_{0-12}, \mathrm{mcg} \cdot \mathrm{h} / \mathrm{mL}\right)$ is 26.7 . In a single dose phase study evaluating the pharmacokinetics of the delayed release tablet versus the oral 
suspension, under fasting conditions, the mean AUC for two posaconazole delayed-release tablet formulations $\left(\mathrm{AUC}_{0-\infty}, \mathrm{mcg} \cdot \mathrm{h} / \mathrm{mL}\right)$ was 11.7 and 11.3 vs. 3.42 for the posaconazole suspension. These values can be affected by gastric motility $[13,15,16,18,19]$. The major elimination route of posaconazole is fecal $(77 \%)$ with a systemic clearance of $421 \mathrm{~mL} / \mathrm{min}$ and a half-life of $24 \mathrm{~h} \mathrm{[14].}$ Posaconazole's major metabolic pathway to produce multiple inactive glucuronides is via phase II UDP-glucuronosyltransferase (UGT) 1A4 [15,20]. Similar to other triazole antifungal agents, posaconazole is classified as a strong CYP450 3A4 (CYP3A4) inhibitor and both a p-glycoprotein substrate and inhibitor [8,21-25].

Since the major route of drug disposition of posaconazole is via the UGT-1A4 pathway, it is postulated that coadministration of an enzyme inducer or inhibitor would alter the pharmacokinetic and/or pharmacodynamic properties of posaconazole [1,15]. The role of UGT in drug metabolism has been previously described [26]. Little is known regarding the effects of posaconazole on other UGT substrates.

CYP3A4 inhibition by posaconazole is thought to be most significant when coadministered with CYP3A4 substrates that undergo extensive first pass metabolism. The interaction is also most likely more significant when the CYP3A4 substrate has a narrow therapeutic window [25]. The FDA approved indications for posaconazole involve the treatment of infections often experienced by seriously ill and/or immunocompromised patients. As such, these patients often are on multiple medications. One such group of patients potentially affected by this interaction are those infected with HIV who are on combination antiretroviral therapy (cART). Of particular concern in this patient population are the potential drug-drug interactions between posaconazole and protease inhibitors (PIs), as well as posaconazole and non-nucleoside reverse transcriptase inhibitors (NNRTIs). The potential interaction between these agents is hypothesized to be due to the CYP3A4 interaction between these particular drug classes. Integrase strand transfer inhibitors (INSTIs) are a relatively new drug class with three available agents on the market. Elvitegravir is currently the only INSTI metabolized via CYP3A4 enzymes, and therefore has the potential to interact with posaconazole [27]. However, this proposed phenomenon has not been studied. The CCR5 antagonist, maraviroc, is both a CYP3A4 and p-glycoprotein substrate. Therefore, it may also interact with posaconazole [28,29]. Drug-drug interactions between nucleoside reverse transcriptase inhibitors (NRTIs) and triazole antifungals appear to be minimal because of the dissimilar drug disposition pathways of these agents. [25,30-32]. Detailed description of these cARTs, including their mechanism of action, has been summarized elsewhere $[28,33]$. The purpose of this review is to examine the literature for reports of clinically significant interactions noted between the various antiretroviral classes when coadministered with posaconazole.

\section{Methods Section}

A computerized literature search was conducted using MEDLINE, EMBASE, Cochrane and International Pharmaceutical Abstract databases to identify studies assessing the potential interaction of posaconazole with HIV antiretrovirals. The following terms were used in various combinations to identify articles published from 1980 to December2013: Posaconazole, SCH 56592, Noxafil, HIV infection, AIDS related opportunistic infection, drug interaction, antiretroviral, protease inhibitors, 
non-nucleoside reverse transcriptase inhibitors, nucleoside reverse transcriptase inhibitors, integrase strand inhibitors, CCR5 antagonists, and fusion inhibitors. Additional relevant articles were identified by reviewing the bibliographies of key articles, reviews, studies, treatment guidelines, and data from Merck and Co., Inc. Two pharmacokinetic studies and three clinical studies involving the use of posaconazole and antiretrovirals were identified in our search. The three clinical studies were reviewed for adverse effects or toxicities that could be due to potential drug interactions between posaconazole and antiretrovirals.

\section{Results and Discussion}

\subsection{Pharmacokinetic Studies on Posaconazole Drug Interactions}

\subsubsection{Posaconazole Plus Atazanavir +/- Ritonavir or Efavirenz}

Krishna et al. evaluated the pharmacokinetic interaction of posaconazole with atazanavir, with and without ritonavir, as well as the pharmacokinetic interaction of posaconazole with efavirenz in a phase I, open-label, randomized, 2-part, crossover study [34]. Part 1 consisted of 12 healthy Caucasian volunteers, with an average age of 45 years old. Group 1 received atazanavir $300 \mathrm{mg}$ daily plus ritonavir $100 \mathrm{mg}$ daily for 7 days followed by atazanavir/ritonavir plus posaconazole $400 \mathrm{mg}$ twice daily for 7 days. Patients then underwent a 28-day washout period after which they were given atazanavir alone for 7 days followed by atazanavir plus posaconazole for 7 days. Group 2 received the same medications in the reverse order. The duration of part 1 of the study was 59 days.

Part 2 of the study was conducted in 17 healthy Caucasian volunteers, with an average age of 43 years old. Participants in group 1 were given efavirenz $400 \mathrm{mg}$ daily for 10 days followed by efavirenz $400 \mathrm{mg}$ daily plus posaconazole $400 \mathrm{mg}$ twice daily for 10 days. Patients then underwent a 28 day washout period, followed by administration of posaconazole $400 \mathrm{mg}$ twice daily alone for 10 days. Group 2 received the same medications in the reverse order. The duration of part 2 of the study was 60 days. For both parts 1 and 2, posaconazole was given after consumption of a standardized high fat meal. Measurement of compliance with treatment regimens was not noted.

The primary comparisons were to determine differences in time to reach maximum concentration $\left(\mathrm{C}_{\max }\right)$ and area under the concentration time curve (AUC), which were analyzed using one-way analysis of variance model. The safety evaluation included collection of adverse events, hematologic monitoring, vital sign measurement, and electrocardiogram (ECG) monitoring. The study was powered at $80 \%$ to detect a difference of $22 \%$ in AUC with 12 subjects per comparison.

Results of part 1 noted coadministration of oral posaconazole $400 \mathrm{mg}$ twice daily and oral atazanavir $300 \mathrm{mg}$ daily for 7 days resulted in increased mean $\mathrm{C}_{\max }$ and mean AUC of atazanavir by 2.6- and 3.7-fold, respectively versus atazanavir alone. Also noted when atazanavir alone was coadministered with posaconazole, there was delayed systemic absorption (from 1.5 to $2 \mathrm{~h}$ ), increased half-life (from 5.85 to $11.1 \mathrm{~h}$ ) and decreased clearance (from $49.7 \mathrm{~L} / \mathrm{h}$ to $9.61 \mathrm{~L} / \mathrm{h}$ ) of atazanavir suggesting a possible enzyme inhibition of CYP3A4 from posaconazole in metabolizing atazanavir at multiple organ sites. When atazanavir plus ritonavir were administered with posaconazole similar results were observed (1.5- and 2.5-fold increases in atazanavir AUC, respectively). Co-administration of atazanavir/ritonavir plus posaconazole led to a 1.5- and 1.8-fold increase of mean $\mathrm{C}_{\max }$ and mean 
AUC of ritonavir, respectively, as compared to the combination of atazanavir/ritonavir alone. The authors attributed this observation potentially to enzyme inhibition of UGT1A1 by atazanavir and additional CYP3A4 inhibition by posaconazole. However, atazanavir and ritonavir did not have an effect on posaconazole's pharmacokinetic parameters implicating these PIs did not share the same metabolic pathway with posaconazole. Approximately $92 \%$ of study subjects reported ocular icterus (92\%) which may relate to benign hyperbilirubinemia, a known side effect of atazanavir. Clinically significant increases in total bilirubin were noted in a total of 11 subjects in this study (range 48-156 umol/L). Baseline bilirubin concentrations were unreported. These incidences may be due in part to the 1.5-2.5 fold increase in atazanavir AUC noted. Other commonly reported adverse effects included pollakiuria (50\%) and abdominal pain $(25 \%)$. This finding collaborates with the study by Smith et al. [35] demonstrating hyperbilirubinemia was a biomarker for high atazanavir concentrations.

Results of part 2 noted coadministration of oral posaconazole and efavirenz resulted in a $45 \%$ and $50 \%$ decrease in mean $\mathrm{C}_{\max }$ and mean AUC of posaconazole, respectively. This suggests an induction of UGT by efavirenz on posaconazole metabolism, as noted by the authors. Conversely, posaconazole had minimal effects on the systemic absorption of efavirenz. Delayed absorption of efavirenz from 2 to $4 \mathrm{~h}$ was noted when administered with posaconazole. The most common adverse events were dizziness (47\%), fatigue (41\%), and headache (41\%).

Based on the results of this two part study, the authors concluded that the potential for increased plasma concentrations of both PIs and NNRTIs could occur when administered with posaconazole. They recommended frequent monitoring for toxicities and adverse events related to these antiviral classes when coadministered with posaconazole. The authors also recommended coadministration of efavirenz and posaconazole be avoided when possible due to the decreased posaconazole exposure noted in this study. This study was of small sample size and short duration with limited confidence intervals and no $p$ values provided to indicate clinical significance. Posaconazole package insert lists atazanavir, ritonavir, and efavirenz as interacting agents based on this study [1].

\subsubsection{Posaconazole Plus Fosamprenavir +/- Ritonavir}

Bruggemann et al. [36] conducted an open-label, three period, cross-over, multiple dose, pharmacokinetic study in healthy volunteers comparing the effects of fosamprenavir alone or fosamprenavir boosted ritonavir on the pharmacokinetics of posaconazole and vice versa. Twenty-four healthy adults, mean age of 36, were administered posaconazole at a dose of $200 \mathrm{mg}$ on day 1, $200 \mathrm{mg}$ twice daily on day 2 , followed by $400 \mathrm{mg}$ twice daily from day 3 forward in combination with fosamprenavir $700 \mathrm{mg}$ twice daily or fosamprenavir/ritonavir $700 \mathrm{mg} / 100 \mathrm{mg}$ twice daily. Medication was given for 10 days followed by a 17-day washout period after which groups switched treatment regimens. The study was powered at $80 \%$ to detect a $20 \%$ difference in posaconazole AUC. The authors noted a statistically significant decrease in exposure to posaconazole when administered with fosamprenavir. The geometric mean ratio (GMR) for posaconazole plus fosamprenavir versus posaconazole alone was 0.77 (90\% CI, 0.68-0.87) for the $\mathrm{AUC}_{0-12}$ and $0.79(90 \% \mathrm{CI}, 0.71-0.89)$ for the $\mathrm{C}_{\max }$. There was no statistically significant difference in the GMR free fraction of posaconazole in the posaconazole only group $(0.988 \%)$ versus the combination group $(1.10 \% ; p=0.177)$. For fosamprenavir plus posaconazole versus fosamprenavir/ritonavir plus posaconazole, the GMR was 
0.35 (90\% CI, 0.32-0.39) for the $\mathrm{AUC}_{0-12}$ and 0.64 (90\% CI; 0.55-0.76) for the $\mathrm{C}_{\max }$. Although no serious adverse events were noted, a total of 141 adverse events were reported. The specifics of the adverse events were not mentioned. The authors concluded that the study showed a "significant, bidirectional pharmacokinetic interaction of posaconazole and fosamprenavir," theorizing the reduction in exposure of posaconazole by fosamprenavir to be due to induction of UGT1A4 or p-glycoprotein, decreased posaconazole absorption, or protein displacement of posaconazole. The small sample size and short duration of this study make it difficult to ascertain the long term effects of coadministration. It should also be noted that the study was conducted in healthy volunteers, making it difficult to extrapolate to the HIV-infected population. Based on the results of this study, it appears boosted fosamprenavir may allow for concomitant administration with posaconazole, however therapeutic drug monitoring of posaconazole should be considered. At this time a target serum concentration of posaconazole has not been established due to a significant interpatient variability in its pharmacokinetics [37-39]. Therefore, concomitant therapy should be avoided if possible.

\subsection{Clinical Trials with Posaconazole in HIV-Infected Patients}

Three studies have evaluated posaconazole for the treatment of oropharyngeal/esophageal candidiasis specifically in HIV infected patients [40-42]. Two of the studies examined specifically treatment of azole-refractory oropharyngeal/esophageal candidiasis in this patient population $[41,42]$. All three studies excluded patients initiating a PI within the last 30 days. In a multicenter, international, phase 3, open-label study, Skiest et al. [42] noted approximately $72 \%$ of participants were receiving antiretroviral therapy. Thirty-eight percent of those were taking NNRTIs, 63\% were taking NRTIs, and 53\% were taking PIs. Patients were given posaconazole suspension for a total of 28 days. Rate of reported adverse events was the primary safety outcome. The most common adverse events were diarrhea and neutropenia, however QT prolongation was noted as a reason for discontinuation for at least one patient in the study. Unfortunately, the authors did not examine the potential link between occurrence of adverse events and the potential that these were precipitated due to a drug interaction, particularly with antiretrovirals. The authors did note the diarrhea and neutropenia in particular could be attributed to the underlying HIV disease, opportunistic infections, or receipt of concomitant medications. Though the percentage of patients on antiretrovirals was noted, which antiretroviral medications they were specifically taking is not given. This makes it difficult to ascertain if any of the adverse events could have been related to a drug interaction with posaconazole.

Vasquez et al. evaluated the efficacy of posaconazole versus fluconazole in the treatment of oropharyngeal candidiasis in 350 HIV infected patients in a multicenter, randomized, evaluator-blinded clinical study [40]. Patients received posaconazole or fluconazole oral suspension for a total of 14 days. Of the 350 patients enrolled, 132 (66\%), were receiving no antiretroviral therapy 29 (8\%), were receiving a NNRTI 117 (32\%) were receiving NRTI, and 85 (24\%) were receiving a PI. Serious adverse events in the posaconazole group were fever, asthenia, abdominal pain, respiratory insufficiency, and lymphadenopathy. The authors did not consider any serious adverse event in the posaconazole group to be related to the study drug and did not examine the potential relationship between occurrences of adverse events related to potential drug interactions, particularly antiretrovirals. 
Vasquez et al. examined the safety and efficacy of posaconazole in the long-term treatment of azole refractory oropharyngeal/esophageal candidiasis in $100 \mathrm{HIV}$ infected patients in a multicenter, international, noncomparative, open-label study [41]. Patients received posaconazole oral suspension for 3 months with the option to continue therapy for up to 12 months if they were considered a clinical responder. Seventy percent of patients were receiving antiretroviral therapy at baseline. Of those, $38 \%$ were receiving NNRTIs, 64\% were receiving NRTIs, and 48\% were receiving PIs. Serious adverse events within the first three months of treatment occurred in four patients and included neutropenia, urticaria, and cardiac failure/sudden death. After 3 months of treatment, serious adverse events occurred in three patients and included elevated hepatic enzymes, jaundice, and neutropenia. The authors suggested that the frequently reported adverse events may be due to HIV infection, HIV-associated opportunistic infections, or concomitant medications. The potential association between adverse events and drug interactions, particularly with antiretrovirals was not explored further. It is plausible that some of the adverse events noted in these patients, particularly the laboratory abnormalities (i.e., hepatic enzyme elevations) could have occurred due to increased antiretroviral serum concentrations associated with concomitant posaconazole administration.

\section{Discussion}

HIV-infected patients often experience many comorbidities as well as opportunistic infections over the course of their lives. With antiretrovirals now recommended for all HIV-infected patients regardless of CD4 count to reduce the risk of disease progression, we can expect to see a greater number of patients on cART. Clinically significant drug-drug interactions between azole antifungals and HIV antiretrovirals have been reported. Guidelines recommend limiting the total daily antifungal dose, monitoring drug concentrations to guide dosage adjustments, monitoring for drug toxicities, and avoidance of the combination when possible depending on the azole antifungal and antiretroviral being coadministered [28]. However, in clinical practice it is often challenging to handle such clinically significant drug-drug interactions when there is little available evidence regarding management. Posaconazole is a newer azole antifungal that except for CYP3A4, has minimal inhibitory activity against CYP450 isoforms CYP1A2, 2C8/9, 2D6, and 2E1, suggesting a smaller drug interaction spectrum than itraconazole, ketoconazole and voriconazole [21]. Its metabolism and elimination are also thought to be mediated primarily via UGT-1A4 and p-glycoprotein [8,21-25]. Regardless of the route of administration, the systemic absorption of posaconazole has been enhanced since the development of a delayed release tablet formulation $[1,18]$.

This article reviews the available literature regarding the potential drug interaction between posaconazole and HIV antiretroviral agents. Posaconazole appears to have the potential to produce clinically significant interactions when used with NNRTIs (i.e., efavirenz), PIs (i.e., atazanavir/ritonavir and fosamprenavir/ritonavir), CCR5 antagonists, and specifically the INSTI, elvitegravir. To date, there are only two available pharmacokinetic studies examining this potential interaction. At this time, it is presumed that the interactions noted within the studies may be a class effect, more studies should be done with other antiretroviral agents. It is postulated that the interaction noted in this study between efavirenz and posaconazole may be more related to induction of UGT, posaconazole's inhibition of CYP3A4 would most likely alter concentrations of the other NNRTIs [1]. Additionally, those NNRTIs 
which also induce CYP3A4, including nevirapine, rilpivirine, efavirenz, and etravirine, may decrease posaconazole concentrations leading to insufficiently treated fungal infections. In vitro, rilpivirine is not a p-glycoprotein substrate, but has been noted to be a weak inducer of CYP3A4, potentially making it less prone to cause drug-drug interactions than other NNRTIs [43]. Since all PIs are both CYP3A4 inhibitors and substrates, we can expect to see similar effects as those noted in the pharmacokinetic studies with atazanavir and fosamprenavir. Ritonavir boosted atazanavir and ritonavir boosted darunavir are both currently recommended as first line agents for the initial treatment of HIV [28]. Since both agents are CYP3A4 inhibitors and substrates, increased concentrations of darunavir could be expected when given with posaconazole similar to those noted in the study by Krishna et al. with atazanavir [34]. Calcagno et al. describe the use of posaconazole for treatment of central nervous system infection with Mucorales and Toxoplama gondii in a young West African HIV infected patient concomitantly given cART with ritonavir boosted darunavir + lamivudine and abacavir. Posaconazole drug concentrations in CSF were subtherapeutic throughout treatment [44]. Currently, guidelines recommend avoiding concomitant administration of atazanavir $+/-$ ritonavir or fosamprenavir and posaconazole. At this time, there is limited data regarding ritonavir-boosted fosamprenavir [28]. The package insert for darunavir makes no recommendation specifically for posaconazole, however does note the potential for increased darunavir concentrations in the presence of other azole antifungals [45].

There are no available studies to date regarding the interaction of posaconazole with the INSTIs or maraviroc. Drug interaction is unlikely to occur with posaconazole and the INSTIs raltegravir or dolutegravir since posaconazole is metabolized by UGT-1A4 whereas the disposition of the two INSTIs is via UGT-1A1 [46-49]. Elvitegravir is metabolized via CYP3A4 enzymes, and therefore has the potential to interact with posaconazole [27]. It is specifically formulated as part of the fixed dose combination Stribild $^{\circledR}$, which also includes cobicistat, a new CYP3A4 inhibitor which serves as a level booster. The package insert recommends the benefit to risk of administration of Stribild ${ }^{\circledR}$ with CYP3A4 inhibitors or inducers be weighed prior to concomitant use [27]. However, this potential interaction of elvitegravir with posaconazole has not been studied at this time.

Despite the lack of available evidence, it is known that the CCR5 antagonist, maraviroc, is a CYP3A4 and p-glycoprotein substrate, so the potential for a clinically significant interaction cannot be ruled out [28,29]. The package insert for maraviroc recommends a dose adjustment of $150 \mathrm{mg}$ twice daily for maraviroc when administered with a CYP3A4 inhibitor and $600 \mathrm{mg}$ twice daily when administered with a CYP3A4 inducer [29]. Based on their pharmacokinetic profiles, most likely there is no significant interaction between posaconazole and either the nucleoside reverse transcriptase inhibitors or the fusion inhibitors. Although the precise interacting mechanism(s) associated with these interactions cannot be fully explained through available pharmacokinetic studies, it has been postulated that CYP3A4, UGT and p-glycoprotein may be involved in the alteration of concentrations of posaconazole and NNRTIs and PIs [50]. Of the three available clinical studies examining the use of posaconazole in HIV infected patients who are on cART, no clinically significant drug interactions were noted [40-42]. However, some toxicities could be attributed to drug interactions between antiretrovirals and posaconazole. Neither antiretroviral or posaconazole serum concentrations were obtained in any of the clinical studies. 
Present opportunistic infection guidelines recommend avoidance of concomitant administration of posaconazole and unboosted fosamprenavir atazanavir $+/-$ ritonavir, and efavirenz. The guidelines also list potential interactions of posaconazole specifically with etravirine, rilpivirine, and elvitegravir, however no dose adjustment is recommended, only that the patient be monitored for signs and symptoms of toxicity [7]. Based on available literature, if concomitant use of posaconazole and one of the interacting agents is medically necessary, frequent monitoring of trough concentrations, adverse drug reactions and dose adjustments may be warranted. At this time therapeutic serum concentrations of posaconazole have not been established due to a significant interpatient variability in its pharmacokinetics [37-39].

Although routine therapeutic drug monitoring of posaconazole has not been widely incorporated into common clinical practice due to lack of outcome studies based on posaconazole concentrations, availability of assays and turnaround time, it is suggested that testing should be performed to ensure adequate drug absorption and prevent drug toxicity from undesirable drug concentrations [51]. Currently, the Department of Health and Human Services antiretroviral guidelines do not recommend therapeutic drug monitoring of antiretroviral agents [28]. Foca et al. [52] recommended therapeutic drug monitoring for unboosted atazanavir in patients with moderate hepatic impairment. For more complex patients receiving concomitant posaconazole and antiretroviral therapy, if available, therapeutic drug monitoring of the antiretrovirals may be useful. Please refer to Table 1 for a summary of recommendations regarding management of this interaction.

Limitations of the studies within this review include relatively small sample sizes, a wide variability of results, exclusion of other potentially interacting medications besides antiretroviral agents and lack of reported drug interaction information between posaconazole and other preferred antiretroviral regimens. It may be difficult to extrapolate these drug interaction data to HIV patients since they may inherently have multiple factors that can alter the pharmacokinetic parameters of a drug $[2,53]$. The evidence of posaconazole's drug interactions in pediatric populations is even more scarce. However, its efficacy and safety profile appears to be similar to the adult population [1]. Present data suggest increased serum concentrations of antiretrovirals, particularly NNRTIs and PIs, as well as decreases in posaconazole AUC with efavirenz. These interactions may increase the risk of adverse effects, toxicities and even the potential for therapeutic failure when coadministered. 
Table 1. Summary of the effects of drug interactions between Posaconazole and HIV Antiretrovirals.

\begin{tabular}{|c|c|c|c|c|c|}
\hline HIV ARV & Effect on ARV Levels & $\begin{array}{l}\text { Effect on } \\
\text { Posaconzole Levels }\end{array}$ & $\begin{array}{l}\text { Potential } \\
\text { Clinical Effects }\end{array}$ & $\begin{array}{l}\text { Mechanism of } \\
\text { Interaction }\end{array}$ & Management \\
\hline \multicolumn{6}{|c|}{ Protease Inhibitors } \\
\hline $\begin{array}{l}\text { Atazanavir } \\
300 \mathrm{mg} \text { daily }[1,34] \\
+ \\
\text { Posaconazole } \\
400 \mathrm{mg} \text { BID }\end{array}$ & $\begin{array}{l}\text { Atazanavir AUC: } \\
\text { increased 268\%; } \\
\text { Cmax: increased 155\% }\end{array}$ & - & $\begin{array}{l}\text { Increased } \\
\text { atazanavir } \\
\text { effects }\end{array}$ & $\begin{array}{l}\text { Inhibition of } \\
\text { CYP450 3A4 } \\
\text { by posaconazole }\end{array}$ & $\begin{array}{l}\text { Dose adjustment not established; } \\
\text { Monitor closely for adverse } \\
\text { effects; recommend therapeutic } \\
\text { drug monitoring of atazanavir } \\
\text { in select patients }\end{array}$ \\
\hline $\begin{array}{l}\text { Atazanavir } \\
300 \text { mg daily } \\
\text { plus ritonavir } \\
100 \text { mg daily }[1,34] \\
+ \\
\text { Posaconazole } \\
400 \text { mg BID }\end{array}$ & $\begin{array}{l}\text { Atazanavir AUC: } \\
\text { increased 146\%; } \\
\text { Cmax: increased 53\% }\end{array}$ & - & $\begin{array}{l}\text { Increased } \\
\text { atazanavir } \\
\text { effects }\end{array}$ & $\begin{array}{l}\text { Inhibition of } \\
\text { CYP450 3A4 } \\
\text { by posaconazole }\end{array}$ & $\begin{array}{l}\text { Dose adjustment not established; } \\
\text { Monitor closely for adverse } \\
\text { effects; recommend therapeutic } \\
\text { drug monitoring of atazanavir } \\
\text { in select patients }\end{array}$ \\
\hline $\begin{array}{l}\text { Fosamprenavir } \\
1400 \mathrm{mg} \text { BID [36] } \\
+ \\
\text { Posaconazole } \\
400 \mathrm{mg} \text { BID }\end{array}$ & $\begin{array}{l}\text { Amprenavir AUC: decreased } \\
65 \% \text {; Cmax: decreased } 36 \% \\
\text { (when compared to } \\
700 \mathrm{mg} / 100 \mathrm{mg} \\
\text { fosamprenavir/ritonavir) }\end{array}$ & $\begin{array}{l}\text { Posaconazole AUC: } \\
\text { decreased } 23 \% \text {; } \\
\text { Cmax decreased } 20 \%\end{array}$ & $\begin{array}{l}\text { Decreased } \\
\text { posaconazole and } \\
\text { fosamprenavir } \\
\text { efficacy }\end{array}$ & - & Do not coadminister \\
\hline $\begin{array}{l}\text { Ritonavir } \\
100 \mathrm{mg} \text { daily }[1,34] \\
+ \\
\text { Posaconazole } \\
400 \mathrm{mg} \text { BID }\end{array}$ & $\begin{array}{l}\text { Ritonavir AUC: } \\
\text { increased } 80 \% \text {; } \\
\text { Cmax: increased } 49 \%\end{array}$ & - & $\begin{array}{l}\text { Possibly } \\
\text { increased } \\
\text { ritonavir effects }\end{array}$ & $\begin{array}{l}\text { Inhibition of } \\
\text { CYP450 3A4 } \\
\text { by posaconazole; } \\
\text { potential inhibition } \\
\text { of UGT 1A1 } \\
\text { by atazanavir }\end{array}$ & Dose adjustment not necessary \\
\hline
\end{tabular}


Table 1. Cont.

\begin{tabular}{|c|c|c|c|c|c|}
\hline HIV ARV & Effect on ARV Levels & $\begin{array}{l}\text { Effect on Posaconzole } \\
\text { Levels }\end{array}$ & $\begin{array}{l}\text { Potential Clinical } \\
\text { Effects }\end{array}$ & $\begin{array}{l}\text { Mechanism of } \\
\text { Interaction }\end{array}$ & Management \\
\hline \multicolumn{6}{|c|}{ NNRTIs } \\
\hline $\begin{array}{l}\text { Efavirenz } 400 \mathrm{mg} \\
\text { daily }[1,34] \\
+ \\
\text { Posaconazole } \\
400 \mathrm{mg} \text { BID }\end{array}$ & $\begin{array}{l}\text { No significant } \\
\text { change }\end{array}$ & $\begin{array}{l}\text { Posaconazole AUC: } \\
\text { decreased } 50 \% \text {; } \\
\text { Cmax: decreased } 45 \%\end{array}$ & $\begin{array}{l}\text { Decreased } \\
\text { posaconazole } \\
\text { effects }\end{array}$ & $\begin{array}{l}\text { Induction of UGT } \\
\text { by efavirenz }\end{array}$ & $\begin{array}{l}\text { Dose adjustment } \\
\text { not established }\end{array}$ \\
\hline $\begin{array}{l}\text { Etravirine [54] } \\
+ \\
\text { Posaconazole } 400 \mathrm{mg} \text { BID }\end{array}$ & $\begin{array}{l}\text { Possibly increased } \\
\text { etravirine levels }\end{array}$ & - & - & - & - \\
\hline \multicolumn{6}{|l|}{ Integrase Inhibitor } \\
\hline $\begin{array}{l}\text { Elvitegravir/ } \\
\text { cobicistat }[7,27] \\
+ \\
\text { Posaconazole } 400 \mathrm{mg} \mathrm{BID}\end{array}$ & $\begin{array}{l}\text { Potentially } \\
\text { increased effects }\end{array}$ & $\begin{array}{l}\text { Potentially } \\
\text { increased effects }\end{array}$ & - & - & $\begin{array}{l}\text { Monitor posaconazole } \\
\text { concentrations with } \\
\text { coadministration }\end{array}$ \\
\hline
\end{tabular}

Adapted from: HIV InSite Database, 2013; Key to Abbreviations: ARV = antiretroviral, AUC = area under the concentration time curve, BID = twice daily, $\mathrm{C}_{\max }=$ maximum plasma concentration, $\mathrm{NA}=$ not available, $\mathrm{QD}=$ daily. 


\section{Conclusions}

Currently available literature points to the potential for clinically significant drug interactions when posaconazole is coadministered with HIV antiretrovirals, specifically NNRTIs and PIs. This review found two pharmacokinetic studies and three clinical trials involving HIV infected patients on antiretrovirals, so the frequency of reported clinically significant drug interactions appears to be small at this time. However, more studies are needed involving a wider range of NNRTIs and PIs to determine the true significance of the interaction. Clinicians should be aware of this potentially significant interaction and avoid coadministration when possible as well as monitor for adverse effects and changes in clinical response to either posaconazole or antiretroviral regimens. When available, consideration should be given to therapeutic drug monitoring of antiretroviral serum concentrations in select patients.

\section{Acknowledgments}

We would like to acknowledge Desiree Eschardies and Samantha Schmidt. Candidates at the Lloyd L. Gregory School of Pharmacy, Palm Beach Atlantic University for reviewing the manuscript.

\section{Author Contributions}

Mara Poulakos (primary author): the primary author was responsible for the development, framework and contributed to the literature search and content of the research paper. Amy Henneman (corresponding author): the corresponding author was responsible for contributing content, handling all communications with the editor, and reviewed and revised the research paper. Simon Leung (co-author): the co-author assisted with the development, literature search, content, reviewed and revised the research paper. All authors contributed proportionally to the sequence of authorship provided in the manuscript.

\section{Conflicts of Interest}

The authors declare no conflict of interest.

\section{References and Notes}

1. Merck and Co., Inc. Noxafil (posaconazole) (Package Insert); Merck and Co., Inc.: Whitehouse Station, NJ, USA, 2013.

2. Pitisuttithum, P.; Negroni, R.; Graybill, J.R.; Bustamante, B.; Pappas, P.; Chapman, S.; Hare, R.S.; Hardalo, C.J. Activity of posaconazole in the treatment of central nervous system fungal infections. J. Antimicrob. Chemother. 2005, 56, 745-755.

3. Xiao, L.; Madison, V.; Chau, A.S.; Loebenberg, D.; Palermo, R.E.; McNicholas, P.M. Three-dimensional models of wild-type and mutated forms of cytochrome P450 14 $\alpha$-sterol demethylases from aspergillus fumigatus and candida albicans provide insights into posaconazole binding. Antimicrob. Agents Chemother. 2004, 48, 568-574.

4. Ghannoum, M.A.; Rice, L.B. Antifungal agents: Mode of action, mechanisms of resistance, and correlation of these mechanisms with bacterial resistance. Clin. Microbiol. 1999, 12, 501-517. 
5. Keating, G.M. Posaconazole. Drugs 2005, 65, 1553-1567.

6. Espinel-Ingroff, A. Comparison of in vitro activities of the new triazole SCH56592 and the echinocandins MK-0991 (L-743,872) and LY303366 against opportunistic filamentous and dimorphic fungi and yeasts. J. Clin. Microbiol. 1998, 36, 2950-2956.

7. Panel on Opportunistic Infections in HIV-Infected Adults and Adolescents. Guidelines for the prevention and treatment of opportunistic infections in HIV-infected adults and adolescents: Recommendations from the Centers for Disease Control and Prevention, the national Institutes of Health, and the HIV Medicine Association of the Infectious Diseases Society of America [55].

8. Sandherr, M.; Maschmeyer, G. Pharmacology and metabolism of voriconazole and posaconazole in the treatment of invasive aspergillosis - review of the literature. Eur. J. Med. Res. 2011, 16, 139-144.

9. Courtney, R.; Wexler, D.; Radwanski, E.; Lim, J.; Laughlin, M. Effect of food on the relative bioavailability of two oral formulations of posaconazole in healthy adults. Br. J. Clin. Pharmacol. 2004, 57, 218-222.

10. Courtney, R.; Pai, S.; Laughlin, M.; Lim, J.; Batra, V. Pharmacokinetics, safety, and tolerability of oral posaconazole administered in single and multiple doses in healthy adults. Antimicrob. Agents Chemother. 2003, 47, 2788-2795.

11. Ezzet, F.; Wexler, D.; Courtney, R.; Krishna, G.; Lim, J.; Laudhlin, M. Oral bioavailability of posaconazole in fasted healthy subjects: Comparison between three regimens and basis for clinical dosage recommendations. Clin. Pharmacokinet. 2005, 44, 211-220.

12. Sansone-Parsons, A.; Krishna, G.; Calzetta, A.; Wexler, D.; Kantesaria, B.; Rosenberg, M.A.; Saltzman, M.A. Effect of a nutritional supplement on posaconazole pharmacokinetics following oral administration to healthy volunteers. Antimicrob. Agents Chemother. 2006, 50, 1881-1883.

13. Krishna, G.; Moton, A.; Ma, L.; Medlock, M.M.; McLeod, J. Pharmacokinetics and absorption of posaconazole oral suspension under various gastric conditions in healthy volunteers. Antimicrob. Agents Chemother. 2009, 53, 958-966.

14. Krishna, G.; Ma, L.; Vickery, D.; Yu, X.; Wu, I.; Power, E.; Beresford, E.; Komjathy, S. Effect of various amounts of liquid nutritional supplement on the pharmacokinetics of posaconazole in healthy volunteers. Antimicrob. Agents Chemother. 2009, 53, 4749-4752.

15. Krieter, P.; Flannery, B.; Musick, T.; Gohdes, M.; Martinho, M.; Courtney, R. Disposition of posaconazole following single dose oral administration in healthy subjects. Antimicrob. Agents Chemother. 2004, 48, 3543-3551.

16. Sansone-Parsons, A.; Krishna, G.; Simon, J.; Soni, P.; Kantesaria, B.; Herron, J.; Stoltz, R. Effects of age, gender, and race/ethnicity on the pharmacokinetics of posaconazole in healthy volunteers. Antimicrob. Agents Chemother. 2007, 51, 495-502.

17. Courtney, R.; Sansone, A.; Smith, W.; Marbury, T.; Statkevich, P.; Martinho, M.; Laughlin, M.; Swan, S. Posaconazole pharmacokinetics, safety and tolerability in subjects with varying degrees of chronic renal disease. J. Clin. Pharmacol. 2005, 45, 185-192.

18. Krishna, G.; Ma, L.; Martinho, M.; O’Mara, E. Single dose phase I study to evaluate the pharmacokinetics of posaconazole in new tablet and capsule formulations relative to oral suspension. Antimicrob. Agents Chemother. 2012, 56, 4196-4201. 
19. Krishna, G.; Ma, L.; Martinho, M.; Preston, R.A.; O’Mara, E. A new solid oral formulation of posaconazole: A randomized clinical trial to investigate rising single- and multiple-dose pharmacokinetics and safety in healthy volunteers. J. Antimicrob. Chemother. 2012, 67, 2725-2730.

20. Ghosal, A.; Hapangama, N.; Yuan, Y.; Achanfuo-Yeboah. J.; Iannucci, R.; Chowdhury, S.; Alton, K.; Patrick, J.E.; Zbaida, S. Identification of human UDP-glucuronosyltransferase enzymes(s) responsible for the glucuronidation of posaconazole (Noxafil). Drug Metab. Dispos. 2004, 32, 267-271.

21. Wexler, D.; Courtney, R.; Richards, W.; Banfield, C.; Lim, J.; Laughlin, M. Effect of posaconazole on cytochrome P450 enzymes: A randomized open-label, two-way crossover study. Eur. J. Pharm. Sci. 2004, 21, 645-653.

22. Krishna, G.; Moton, A.; Ma, L.; Savant, I.; Martinho, M.; Seiberling, M.; McLeod, J. Effects of oral posaconazole on the pharmacokinetic properties of oral and intravenous midazolam: A phase I, randomized, open-label, crossover study in healthy volunteers. Clin. Ther. 2009, 31, 286-298.

23. Saad, A.H.; DePestel, D.D.; Carver, P.L. Factors influencing the magnitude and clinical significance of drug interactions between azole antifungals and select immunosuppressants. Pharmacotherapy 2006, 26, 1730-1744.

24. U.S. Food and Drug Administration. Drug Development and Drug Interactions: Table of Substrates, Inhibitors and Inducers. Available online: http://www.fda.gov/drugs/developmentapprovalprocess/ developmentresources/druginteractionslabeling/ucm093664.htm (accessed on 5 December 2013).

25. Lipp, H.P. Clinical pharmacodynamics and pharmacokinetics of the antifungal extended-spectrum triazole posaconazole: An overview. Br. J. Clin. Pharmacol. 2010, 70, 471-480.

26. Rowland, A.; Miners, J.O.; Mackenzie, P.I. The UDP-Glucuronosyltransferases: Their role in drug metabolism and detoxification. Int. J. Biochem. Cell Biol. 2013, 45, 1121-1132.

27. Gilead Sciences, Inc. Stribild (elvitegravir, cobicistat, emtricitabine, tenofovir) (Package Insert); Gilead Sciences, Inc.: Foster City, CA, USA, 2012.

28. Guidelines for the use of antiretroviral agents in HIV-1 infected adults and adolescents. Available online: http://aidsinfo.nih.gov/ContentFiles/AdultandAdolescentGL.pdf (accessed on 5 December 2013).

29. ViiV Healthcare. Selzentry (maraviroc) (Package Insert); ViiV Healthcare: Research Triangle Parck, NC, USA, 2013.

30. Safrin, S. Antiviral Agents. In Basic and Clinical Pharmacology, 12nd ed.; Katzung, B.G., Masters, S.B., Trevor, A.J., Eds.; McGraw-Hill: New York, NY, USA, 2012; Available online: http://www.accesspha (accessed on 5 December 2013).

31. Warnke, D.; Barreto, J.; Temesgen, Z. Antiretroviral drugs. J. Clin. Pharmacol. 2007, 47, 1570-1579. Available online: http://rmacy.com/content.aspx?aID=55829973 (accessed on 5 December 2013).

32. Piacenti, F.J. An update and review of antiretroviral therapy. Pharmacotherapy 2006, 26, 1111-1133.

33. Toronto General Hospital. Immunodeficiency Clinic Website. Available online: http://hivclinic.ca (accessed on 5 December 2013).

34. Krishna, G.; Moton, A.; Ma, L.; Martinho, M.; Seiberling, M.; McLeod, J. Effects of oral posaconazole on the pharmacokinetics of atazanavir alone and with ritonavir or with efavirenz in healthy adult volunteers. J. Acquir. Immune Defic. Syndr. 2009, 51, 437-444. 
35. Smith, D.E.; Jeganathan, S.; Ray, J. Atazanavir plasma concentrations vary significantly between patients and correlate with increased serum bilirubin concentrations. HIV Clin. Trials. 2006, 7 , 34-38.

36. Bruggemann, R.; van Luin, M.; Colbers, E.P.; van den Dungen, M.W.; Pharo, C.; Schouwenberg, B.J.; Burger, D.M. Effect of posaconazole on the pharmacokinetics of fosamprenavir and vice versa in healthy volunteers. J. Antimicrob. Chemother. 2010, 65, 2188-2194.

37. Andes, D.; Pascual, A.; Marchetti, O. Antifungal therapeutic drug monitoring: Established and emerging indications. Antimicrob. Agents Chemother. 2009, 53, 24-34.

38. Gubbins, P.O.; Krishna, G.; Sansone-Parsons, A.; Penzak, S.R.; Dong, L.; Martinho, M.; Anaissie, E.J. Pharmacokinetics and safety of oral posaconazole in neutropenic stem cell transplant recipients. Antimicrob. Agents Chemother. 2006, 50, 1993-1999.

39. Ullmann, A.J.; Cornely, O.A.; Burchardt, A.; Hachem, R.; Kontoyiannis, D.P.; Töpelt, K.; Courtney, R.; Wexler, D.; Krishna, G.; Martinho, M.; et al. Pharmacokinetics, safety, and efficacy of posaconazole in patients with persistent febrile neutropenia or refractory invasive fungal infection. Antimicrob. Agents Chemother. 2006, 50, 658-666.

40. Vazquez, J.A.; Skiest, D.J.; Nieto, L.; Northland, R.; Sanne, I.; Gogate, J.; Greaves, W.; Isaacs, R. A multicenter randomized trial evaluating posaconazole versus fluconazole for the treatment of oropharyngeal candidiasis in subjects with HIV/AIDS. Clin. Infect. Dis. 2006, 42, 1179-1186.

41. Vazquez, J.A.; Skiest, D.J.; Tissot-Dupont, H.; Lennox, J.L.; Boparai, N.; Isaacs, R. Safety and efficacy of posaconazole in the long-term treatment of azole-refractory oropharyngeal and esophageal candidiasis in patients with HIV infection. HIV Clin. Trials. 2007, 8, 86-97.

42. Skiest, D.J.; Vazquez, J.A.; Anstead, G.M.; Graybill, J.R.; Reynes, J.; Ward. D.; Hare, R.; Boparai, N.; Isaacs, R. Posaconazole for the treatment of azole-refractory oropharyngeal and esophageal candidiasis in subjects with HIV infection. Clin. Infect. Dis. 2007, 44, 607-614.

43. Weiss, J.; Haefeli, W.E. Potential of the novel antiretroviral drug rilpivirine to modulate the expression and function of drug transporters and drug-metabolising enzymes in vitro. Int. J. Antimicrob. Agents 2013, 41, 484-487.

44. Calcagno, A.; Baietto, L.; de Rosa, F.; Tettoni, M.C.; Libanore, V.; Bertucci, R.; D’Avolio, A.; di perri, G. Posaconazole cerebrospinal concentrations in an HIV-infected patient with brain mucormycosis. J. Antimicrob. Chemother. 2011, 66, 224-225.

45. Janssen Pharmaceuticals, Inc. Prezista (darunavir) (Package Insert); Janssen Pharmaceuticals, Inc.: Titusville, NJ, USA, 2006.

46. Merck Sharp \& Dohme Corp. Isentress (raltegravir) (Package Insert); Merck Sharp \& Dohme Corp.: Whitehouse Station, NJ, USA, 2011.

47. Hashiguchi, Y.; Hamada, A.; Shinohara, T.; Tsuchiya, K.; Jono, H.; Saito, H. Role of P-Glycoprotein in the efflux of raltegravir from human intestinal cells and CD4+ T-cells as an interaction target for anti-HIV agents. Biochem. Biophys. Res. Commun. 2013, 439, 221-227.

48. Kassahun, K.; McIntosh, I.; Cui, D.; Hreniuk, D.; Merschman, S.; Lasseter, K.; Azrolan, N.; Iwamoto, M.; Wagner, J.A.; Wenning, L.A. Metabolism and disposition in humans of raltegravir (MK-0518), an anti-AIDS drug targeting the human immunodeficiency virus 1 integrase enzyme. Drug Metab. Dispos. 2007, 35, 1657-1663. 
49. Reese, M.J.; Savina, P.M.; Generaux, G.T.; Tracey, H.; Humphreys, J.E.; Kanaoka, E.; Webster, L.O.; Harmon, K.A.; Clarke, J.D.; Polli, J.W. In vitro investigations into the roles of drug transporters and metabolizing enzymes in the disposition and drug interactions of dolutegravir, a HIV integrase inhibitor. Drug Metab. Dispos. 2013, 41, 353-361.

50. Leung, S.; Poulakos, M. Drug interaction profile of posaconazole. Int. J. Allied Health Sci. Pract. 2008, 6, 1-8.

51. Thompson, G.R.; Rinaldi, M.G.; Pennick, G.; Dorsey, S.A.; Patterson, T.F.; Lewis, J.S. Posaconazole therapeutic drug monitoring: A reference laboratory experience. Antimicrob. Agents Chemother. 2009, 53, 2223-2224.

52. Foca, E.; Ripamonti, D.; Motta, D.; Torti, C. Unboosted atazanavir for treatment of HIV infection. Drugs 2012, 72, 1161-1173.

53. Welage, L.S.; Carver, P.L.; Revankar, S.; Pierson, C.; Kauffman, C.A. Alteraction sin gastric acidity in patients infected with human immunodeficiency virus. Clin. Infect. Dis. 1995, 21, $1431-1438$.

54. Tibotec Therapeutics. Intelence (etravirine) (Package Insert); Tibotec Therapeutics: Raritan, NJ, USA, 2010.

55. AIDSinfo. Clinical Guidelines Portal. Available online: http://aidsinfo.nih.gov/guidelines (accessed on 5 December 2013).

(C) 2014 by the authors; licensee MDPI, Basel, Switzerland. This article is an open access article distributed under the terms and conditions of the Creative Commons Attribution license (http://creativecommons.org/licenses/by/3.0/). 